\title{
Unsteady Heat Transfer In a Gas Mixture
}

\author{
Alexey Polikarpov ${ }^{1,2, a}$, Minh Tuan $\mathrm{Ho}^{3}$ and Irina Graur ${ }^{4}$ \\ ${ }^{1}$ Ural Federal University, Physics Department, Lenina str. 51, 620000, Ekaterinburg, Russia \\ ${ }^{2}$ Kutateladze Institute of Thermophysics SB RAS, Lavrentyev Ave., Novosibirsk, 630090, Russia \\ ${ }^{3}$ James Weir Fluids Laboratory, Department of Mechanical and Aerospace Engineering, University of Strathclyde, \\ Glasgow G1 1 XJ, UK \\ ${ }^{4}$ Aix-Marseille University, rue Enrico Fermi 5, 13013, Marseille, France
}

\begin{abstract}
The time-dependent problem of heat transfer between two parallel plates in a binary gas mixture was investigated on the basis of numerical solution of BGK type kinetic model equation with collision integral taken in the form of McCormack model. The time evolution of the normal heat flux was simulated and time needed to reach the steady state conditions was obtained.
\end{abstract}

\section{Introduction}

Heat and mass transfer phenomena in a gas near a heated or cooled surface play a key role in large number of technological processes. In addition, in most of these processes the transport phenomena are essentially time-dependent. Actual tendency of many technical devices miniaturization leads to increase of a ratio between the molecular mean free path and the characteristic scale of a device (this ratio is called Knudsen number). If the Knudsen number becomes larger than one a gas inside this device must be considered as rarefied and its behavior must be simulated on the basis of the Boltzmann equation.

The heat transfer in a rarefied gas mixture confined between two parallel plates was intensively studied during the last decades, see Refs. [1,2,3]. One of these works is devoted to the case of steady state regime [1]. The transient behaviour in collisionless regime was studied in Ref. [2], while the oscillatory boundary conditions were stated in Ref. [3]. Apparently, only a single work is devoted to the consideration of unsteady heat transfer in a binary gas mixture confined between concentric cylinders [4].

Here the fundamental problem of the transient heat transfer through a gas mixture between two parallel plates, induced by the instantaneous temperature rise and drop of the upper and lower plate, respectively, is simulated on the basis of the McCormack kinetic model [5].

\section{Problem Statement}

Let us consider two parallel infinite plates maintained at different temperatures. The temperature of the upper plate is larger than that of lower and the temperature difference between the plates is equal

\footnotetext{
${ }^{\text {a }}$ Corresponding author : Alexey/Polikarpov@urfu.ru
} 
to $\Delta \mathrm{T}$. The plates are spaced at a distance $\mathrm{H}$. The gap between the plates is filled by a rarefied gaseous mixture. The time evolution of the occurring heat flux is the goal of discussion.

$$
T_{0}+\Delta T
$$

$$
T_{0}-\Delta T
$$

Figure 1. Task geometry

Initially a gas mixture between two parallel plates is in an equilibrium state. Then, the temperature of one plate increases instantaneously and the relaxation of this system to its new equilibrium state is observed. The time evolution of the heat flux is investigated in a wide range of governing parameters: Knudsen number, gas mixture composition and species concentration. The cases of the mixtures with similar (Ne-Ar) and different (He-Ar) molecular masses are considered. The time-dependent linearized kinetic equation for a binary gas mixture is solved numerically

$$
\frac{\partial h_{\alpha}}{\partial t}+v_{y} \frac{\partial h_{\alpha}}{\partial y}=\sum_{\beta=1,2} L_{\alpha \beta} h_{\beta}, \alpha=1,2
$$

where $L_{\alpha \beta} h$ is the linearized collision term. The McCormack model [5] is used here for the calculations of the collision term.

The discrete-velocity method is used to solve numerically the McCormack model equation. The discrete velocity set was taken according to roots of Gauss-Hermite polynomials. The second order spatial discretization with explicit time integration is used. The iteration procedure was used to calculate the unknown values of physical quantities, particularly the value of normal heat flux.

The governing parameters for this task are:

1. The molar concentration of the mixture, which is defined as

$$
C=\frac{n_{1}}{n_{1}+n_{2}}
$$

$n_{\alpha}(\alpha=1,2)$ is the number density of specie $\alpha$.

2. The rarefaction parameter which is defined as

$$
\delta=\frac{p H}{\mu v_{0}}
$$

Here $p$ is the gas mixture pressure, $v_{0}$ is the most probable molecular speed of a gas mixture:

$$
v_{o}=\sqrt{\frac{2 k T_{0}}{m}}
$$

$m=C_{0} m_{1}+\left(1-C_{0}\right) m_{2}$ ( $m_{1}$ is the mass of the lighter component), $\mu$ is the gas mixture viscosity, $\mathrm{k}$ is the Boltzmann constant.

The time evolution of normal heat flux is given in nondimensional form

$$
q_{y}=\frac{q_{y}^{\prime}}{p v_{0}} \frac{T_{0}}{\Delta T}
$$


The upper superscript defines the dimensional value.

\section{Results and Discussion}

The dependence of the heat flux on the values of concentration parameter $\mathrm{C}_{0}$ and rarefaction parameter $\delta$ are studied numerically. The time needed to reach the steady-state conditions is estimated. Figures 2 and 3 show the time evolution of heat flux in the center of the gap between the plates for three values of concentration $\mathrm{C}_{0}$ and $\delta=10$ (near continuum regime). Figure 2 shows the case of quite dissimilar masses of species components (He-Ar), while Figure 3 illustrates the case of similar masses of species components (Ne-Ar).

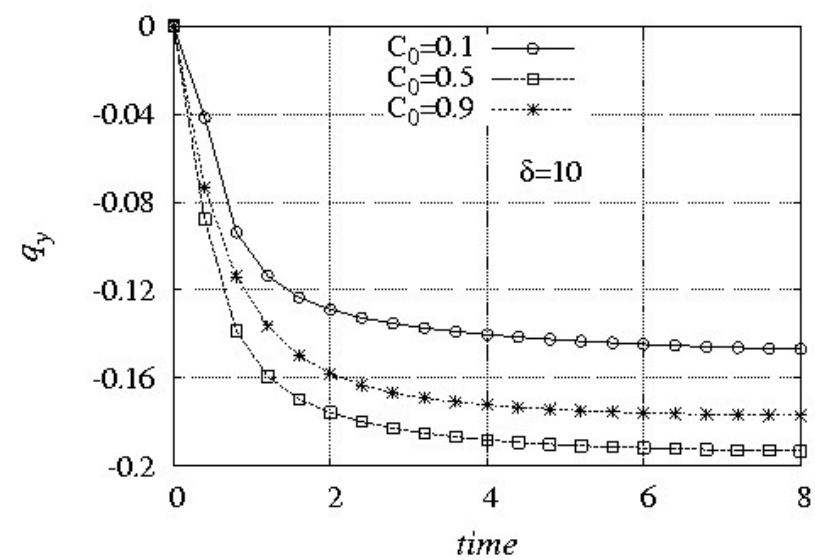

Figure 2. Time evolution of normal heat flux in the center of the gap for mixture He-Ar. Time unit on abscissa axis corresponds to one thousand discrete time steps $\Delta$ t.

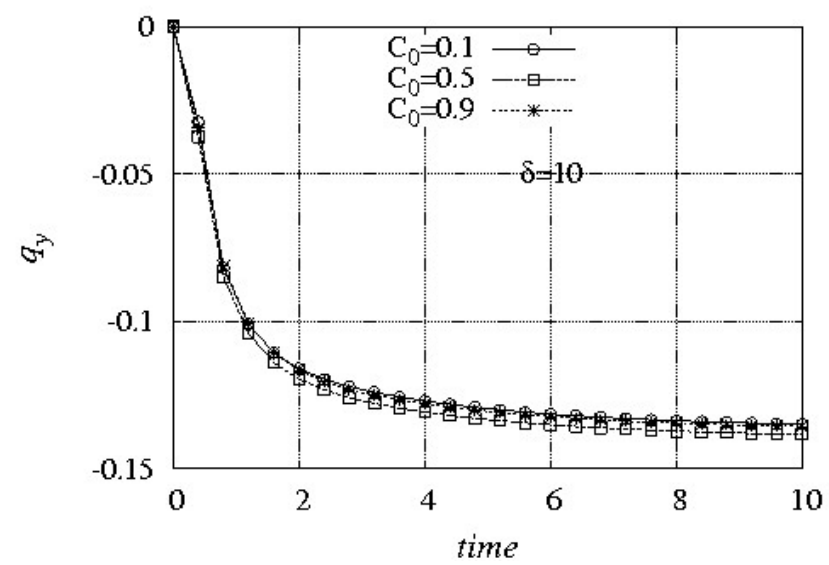

Figure 3. Time evolution of normal heat flux in the center of the gap for mixture Ne-Ar. Time unit on abscissa axis corresponds to one thousand discrete time steps $\Delta$ t.

Due to sudden change of the temperatures of the both plates the value of a heat flux starts to decreases and it changes from «0» value to its steady state value. The results were obtained for three values of concentration $\mathrm{C}_{0}=0.1,0.5,0.9$, for rarefaction parameter $\delta=10$ and two types of a gas mixture. As it follows from Figures 2 for He-Ar mixture the normal heat flux relaxation speed $\left(\mathrm{dq}_{\mathrm{\gamma}} / \mathrm{dt}\right)$ depends on the value of concentration parameter $\mathrm{C}_{0}$, it reaches the maximum value for $\mathrm{C}_{0}=0.5$ (Figure 2 ) and then drop for larger values of concentration parameter. The values of dimensional normal heat 
flux takes a maximum also for $\mathrm{C}_{0}=0.5$. The time necessary to reach the steady state value has the order equivalent to 10 reference time. Figures 2,3 shows the considerable change of heat flux values happened in the earlier time interval less than two values of reference time $\left(\mathrm{H} / \mathrm{v}_{0}\right)$ and then the curves approach the steady-state limit. The values of heat flux values for Ne-Ar mixture do not depend on the concentration parameter. Independently of the composition of the gas mixture the time needed to reach the steady state values has approximately the same order.

\section{Acknowledgment}

The study was supported by the Ministry of Education and Science of the Russian Federation (project identifier RFMEFI61614X0016).

\section{References}

1. Sone Y. J. Phys. Soc. Jpn., 20(2):222 (1965).

2. D C Wadsworth, D A Erwin, and E Ph Muntz. J. of Fluid Mech., 248(1):219, (1993)

3. A Manela and N. G. Hadjiconstantinou. Phys. Fluids, 20 (11):117104, (2008).

4. M Vargas, S Stefanov, and V Roussinov. International Journal of Heat and Mass Transfer, 59:302, (2013).

5. McCormack F. J. Phys. Fluids 16, 12. (1973). 